\title{
"Shaping" the Future of Molecular Weight Distributions in Anionic Polymerization
}

\author{
Veronika Kottisch, Dillon T. Gentekos, and Brett P. Fors \\ Cornell University, Ithaca, NY 14853
}

Supporting Information

\section{General Reagent Information}

All reactions were performed in an Unilab MBraun Glovebox with a nitrogen atmosphere. Styrene (99+\%, Sigma Aldrich), isoprene (>99+\%, Sigma Aldrich), and cyclohexane (Fischer Scientific, ACS Grade) were dried over calcium hydride $\left(\mathrm{CaH}_{2}\right)$ (ACROS organics, 93\% extra pure, 0-2 mm grain size) for $12 \mathrm{~h}$. Styrene was vacuum transferred degassed by three freeze-pump-thaw cycles. Cyclohexane and isoprene were distilled under nitrogen and degassed by vigorously sparging with nitrogen for 30 minutes or three freeze-pump-thaw cycles, respectively. Sec-butyllithium (Sigma Aldrich, 1.4 M in cyclohexane) and isopropanol (anhydrous, 99.5\%) were used as received.

\section{General Analytical Information}

All polymer samples were analyzed using a Tosoh EcoSec HLC 8320GPC system with two SuperHM-M columns in series at a flow rate of $0.350 \mathrm{~mL} / \mathrm{min}$. THF was used as the eluent and all number-average molecular weights $\left(M_{n}\right)$, weight-average molecular weights $\left(M_{w}\right)$, dispersities $(\bigoplus)$, asymmetry factors $\left(A_{s}\right), M_{z}$ and $M_{z+1}$ were calculated from refractive index chromatograms against TSKgel polystyrene standards. For the data in Table S8 a MiniDawn Treos MALLS detector was used. Conversions were determined on a Varian $400 \mathrm{MHz}$ NMR spectrometer in $\mathrm{CDCl}_{3}$. Tensile properties of compression molded block copolymer samples were analyzed by dynamic mechanical analysis using a TA Instruments DMA Q800 Dynamic Mechanical Thermal Analysis (DMTA) instrument. 


\section{General Procedure for Styrene Polymerizations}

A $20 \mathrm{~mL}$ scintillation vial equipped with a magnetic stirrer was flame dried, brought into the glove box, and charged with $6.9 \mathrm{~mL}$ of cyclohexane and $0.8 \mathrm{~mL}$ of styrene $(6.98$ $\mathrm{mmol})$. A sec-butyllithium stock solution in cyclohexane $(0.16 \mathrm{M})$ was prepared for the reactions and a total volume of $360 \mu \mathrm{L}$ of the solution was drawn into a $1 \mathrm{~mL}$ syringe and then mounted onto a New Era NE-4000 Double Syringe Pump. The pump was programmed according to the appropriate rate profile, which would dispense a total volume of $320 \mu \mathrm{L}(0.0512 \mathrm{mmol}$ of $s \mathrm{BuLi})$ of the initiator solution. Once the needle was submerged into the reaction mixture, the addition program was started. The reaction turned slowly bright orange over the course of the addition due to the formation of the polystyrylanion. The reaction was allowed to reach full conversion and was quenched with excess isopropanol until the solution was clear and colorless. The polymer was isolated by removing the solvent under vacuum overnight to afford a white solid.

Note: For the $80 \mathrm{~min}, 100 \mathrm{~min}$, and $120 \mathrm{~min}$ additions of exponentially increasing rates a $0.0533 \mathrm{M}$ initiator solution was prepared. The total initiator solution volume and cyclohexane volume were adjusted to $960 \mu \mathrm{L}(0.0512 \mathrm{mmol})$ and $6.3 \mathrm{~mL}$, respectively. 
Procedure for Figure 2a-d: Constant Addition Rate Profiles

The synthesis was performed according to the general procedure and the New Era NE4000 Double Syringe Pump was programmed to the following addition rate profiles for the constant rates shown in Table S1.

Table S1. Constant Rate Addition Profiles

$\begin{array}{ccc}\begin{array}{c}\text { Addition Time } \\ (\mathbf{m i n})\end{array} & \begin{array}{c}\text { Rate } \\ (\boldsymbol{\mu L} / \mathrm{h})\end{array} & \begin{array}{c}\text { Total Volume } \\ (\boldsymbol{\mu L})\end{array} \\ 20 & 960 & 320 \\ 40 & 480 & 320 \\ 60 & 320 & 320 \\ 80 & 240 & 320 \\ 100 & 192 & 320 \\ 120 & 160 & 320\end{array}$

Procedure for Figure 2e-h: Linearly Ramped Addition Rate Profiles

The synthesis was performed according to the general procedure. All linearly increasing addition rate profiles were programmed as a sequence of 20 step increments with each step corresponding to a phase in the New Era NE-4000 Double Syringe Pump program. See Table S2 for detailed rates and volumes. 
Table S2. Linearly Ramped Rate Addition Profile

\begin{tabular}{|c|c|c|c|c|c|c|c|c|}
\hline \multirow[t]{2}{*}{ Step \# } & \multicolumn{7}{|c|}{ Rate $(\mu L / h)$} & \multirow{2}{*}{$\begin{array}{c}\text { Volume per } \\
\text { Step }(\mu L)\end{array}$} \\
\hline & $20 \mathrm{~min}$ & $30 \mathrm{~min}$ & $40 \mathrm{~min}$ & $50 \mathrm{~min}$ & $60 \mathrm{~min}$ & $80 \mathrm{~min}$ & $100 \mathrm{~min}$ & \\
\hline 1 & 167 & 111 & 83 & 67 & 56 & 42 & 33 & 2.8 \\
\hline 2 & 250 & 167 & 125 & 100 & 83 & 62 & 50 & 4.2 \\
\hline 3 & 334 & 223 & 167 & 134 & 111 & 83 & 67 & 5.6 \\
\hline 4 & 417 & 278 & 209 & 167 & 139 & 104 & 84 & 7.0 \\
\hline 5 & 501 & 334 & 250 & 200 & 167 & 125 & 100 & 8.3 \\
\hline 6 & 584 & 390 & 292 & 234 & 195 & 146 & 117 & 9.7 \\
\hline 7 & 668 & 445 & 334 & 267 & 223 & 167 & 134 & 11.1 \\
\hline 8 & 751 & 501 & 376 & 301 & 250 & 188 & 150 & 12.5 \\
\hline 9 & 835 & 557 & 417 & 334 & 278 & 209 & 167 & 13.9 \\
\hline 10 & 918 & 612 & 459 & 367 & 306 & 230 & 184 & 15.3 \\
\hline 11 & 1002 & 668 & 501 & 401 & 334 & 250 & 200 & 16.7 \\
\hline 12 & 1085 & 724 & 543 & 434 & 362 & 271 & 217 & 18.1 \\
\hline 13 & 1169 & 779 & 584 & 468 & 390 & 292 & 234 & 19.5 \\
\hline 14 & 1252 & 835 & 626 & 501 & 417 & 313 & 250 & 20.9 \\
\hline 15 & 1336 & 890 & 668 & 534 & 445 & 334 & 267 & 22.3 \\
\hline 16 & 1419 & 946 & 710 & 568 & 473 & 355 & 284 & 23.7 \\
\hline 17 & 1503 & 1002 & 751 & 601 & 501 & 376 & 301 & 25.0 \\
\hline 18 & 1586 & 1057 & 793 & 634 & 529 & 397 & 317 & 26.4 \\
\hline 19 & 1670 & 1113 & 835 & 668 & 557 & 417 & 334 & 27.8 \\
\hline 20 & 1753 & 1169 & 877 & 701 & 584 & 438 & 351 & 29.2 \\
\hline
\end{tabular}




\section{Procedure for Figure 2i-I: Exponentially Ramped Addition Rate Profiles}

The synthesis was performed according to the general procedure. All exponentially increasing addition profiles were programmed as a sequence of 20 step increments with each step corresponding to a phase in the New Era NE-4000 Double Syringe Pump program. See Table S3 for detailed rates and volumes. For 80, 100, and 120 min long exponentially ramped additions, the total addition volume had to adjusted to $960 \mu \mathrm{L}$ due to a more dilute initiator solution ( $0.0533 \mathrm{M})$ (Table S4).

Table S3. Exponentially Ramped Rate Addition Profile for 20 - 60 min

\begin{tabular}{|c|c|c|c|c|}
\hline \multirow[t]{2}{*}{ Step \# } & \multicolumn{3}{|c|}{ Rate ( $\mu L / h)$} & \multirow{2}{*}{$\begin{array}{c}\text { Volume per } \\
\text { Step }(\mu L)\end{array}$} \\
\hline & $20 \mathrm{~min}$ & $40 \mathrm{~min}$ & $60 \mathrm{~min}$ & \\
\hline 1 & 9.2 & 4.6 & 3.1 & 0.2 \\
\hline 2 & 12.9 & 6.4 & 4.3 & 0.2 \\
\hline 3 & 18.0 & 9.0 & 6.0 & 0.3 \\
\hline 4 & 25.2 & 12.6 & 8.4 & 0.4 \\
\hline 5 & 35.3 & 17.7 & 11.8 & 0.6 \\
\hline 6 & 49.4 & 24.7 & 16.5 & 0.8 \\
\hline 7 & 69.2 & 34.6 & 23.1 & 1.2 \\
\hline 8 & 96.9 & 48.5 & 32.3 & 1.6 \\
\hline 9 & 135.6 & 67.8 & 45.2 & 2.3 \\
\hline 10 & 189.9 & 95.0 & 63.3 & 3.2 \\
\hline 11 & 265.9 & 132.9 & 88.6 & 4.4 \\
\hline 12 & 372.2 & 186.1 & 124.1 & 6.2 \\
\hline 13 & 521.1 & 260.6 & 173.7 & 8.7 \\
\hline 14 & 729.5 & 364.8 & 243.2 & 12.2 \\
\hline 15 & 1021 & 510.7 & 340.4 & 17.0 \\
\hline 16 & 1430 & 715.0 & 476.6 & 23.8 \\
\hline 17 & 2002 & 1001 & 667.3 & 33.4 \\
\hline 18 & 2803 & 1401 & 934.2 & 46.7 \\
\hline 19 & 3924 & 1962 & 1308 & 65.4 \\
\hline 20 & 5493 & 2747 & 1831 & 91.6 \\
\hline
\end{tabular}


Table S4. Exponentially Ramped Rate Addition Profile for 80 - 120 min

\begin{tabular}{|c|c|c|c|c|}
\hline \multirow[t]{2}{*}{ Step \# } & \multicolumn{3}{|c|}{$\begin{array}{l}\text { Rate } \\
(\mu \mathrm{L} / \mathrm{h})\end{array}$} & \multirow[t]{2}{*}{$\begin{array}{c}\text { Volume per } \\
\text { Step }(\mu L)\end{array}$} \\
\hline & $80 \mathrm{~min}$ & $100 \mathrm{~min}$ & $120 \mathrm{~min}$ & \\
\hline 1 & 6.9 & 5.5 & 4.6 & 0.5 \\
\hline 2 & 9.7 & 7.7 & 6.4 & 0.6 \\
\hline 3 & 13.5 & 10.8 & 9.0 & 0.9 \\
\hline 4 & 18.9 & 15.1 & 12.6 & 1.3 \\
\hline 5 & 26.5 & 21.1 & 17.7 & 1.8 \\
\hline 6 & 37.1 & 29.7 & 24.7 & 2.5 \\
\hline 7 & 51.9 & 41.5 & 34.6 & 3.5 \\
\hline 8 & 72.7 & 58.1 & 48.4 & 4.8 \\
\hline 9 & 101.7 & 81.4 & 67.8 & 6.8 \\
\hline 10 & 142.4 & 113.9 & 95.0 & 9.5 \\
\hline 11 & 199.4 & 159.5 & 132.9 & 13.3 \\
\hline 12 & 279.1 & 223.3 & 186.1 & 18.6 \\
\hline 13 & 390.8 & 312.6 & 260.6 & 26.1 \\
\hline 14 & 547.1 & 437.7 & 364.8 & 36.5 \\
\hline 15 & 765.9 & 612.7 & 510.7 & 51.1 \\
\hline 16 & 1072 & 857.8 & 715.0 & 71.5 \\
\hline 17 & 1501 & 1201 & 1001 & 100.1 \\
\hline 18 & 2102 & 1681 & 1401 & 140.1 \\
\hline 19 & 2942 & 2354 & 1962 & 196.2 \\
\hline 20 & 4119 & 3295 & 2747 & 274.6 \\
\hline
\end{tabular}


Procedure for Figure 5: Bell-Shaped Addition Rate Profiles

The synthesis was performed according to the general procedure. All bell-shaped addition profiles were programmed as a sequence of 20 step increments with each step corresponding to a phase in the New Era NE-4000 Double Syringe Pump program. See Table S5 for detailed rates and volumes.

Table S5: Bell-Shaped Rate Addition Profile

\begin{tabular}{|c|c|c|c|c|c|c|}
\hline \multirow[t]{2}{*}{ Step \# } & \multicolumn{5}{|c|}{ Rate ( $\mu L / h)$} & \multirow{2}{*}{$\begin{array}{c}\text { Volume per } \\
\text { Step }(\mu L)\end{array}$} \\
\hline & $20 \mathrm{~min}$ & $40 \mathrm{~min}$ & $60 \mathrm{~min}$ & $80 \mathrm{~min}$ & $100 \mathrm{~min}$ & \\
\hline 1 & 25.6 & 17.0 & 12.8 & 10.2 & 8.5 & 0.9 \\
\hline 2 & 76.7 & 51.1 & 38.3 & 30.7 & 25.6 & 2.6 \\
\hline 3 & 153.4 & 102.2 & 76.7 & 61.3 & 51.1 & 5.1 \\
\hline 4 & 255.6 & 170.4 & 127.8 & 102.2 & 85.2 & 8.5 \\
\hline 5 & 383.4 & 255.6 & 127.8 & 153.4 & 127.8 & 12.8 \\
\hline 6 & 536.8 & 357.8 & 191.7 & 214.7 & 178.9 & 17.9 \\
\hline 7 & 766.8 & 511.2 & 268.4 & 306.7 & 255.6 & 25.6 \\
\hline 8 & 945.7 & 630.5 & 383.4 & 378.3 & 315.2 & 31.5 \\
\hline 9 & 1074 & 715.7 & 472.9 & 429.4 & 357.8 & 35.8 \\
\hline 10 & 1150 & 766.8 & 536.8 & 460.1 & 383.4 & 38.3 \\
\hline 11 & 1074 & 715.7 & 575.1 & 429.4 & 357.8 & 35.8 \\
\hline 12 & 945.7 & 630.5 & 536.8 & 378.3 & 315.2 & 31.5 \\
\hline 13 & 766.8 & 511.2 & 472.9 & 306.7 & 255.6 & 25.6 \\
\hline 14 & 536.8 & 357.8 & 383.4 & 214.7 & 178.9 & 17.9 \\
\hline 15 & 383.4 & 255.6 & 268.4 & 153.4 & 127.8 & 12.8 \\
\hline 16 & 255.6 & 170.4 & 191.7 & 102.2 & 85.2 & 8.5 \\
\hline 17 & 153.4 & 102.2 & 76.7 & 61.3 & 51.1 & 5.1 \\
\hline 18 & 76.7 & 51.1 & 38.3 & 30.7 & 25.6 & 2.6 \\
\hline 19 & 25.6 & 17.0 & 12.8 & 10.2 & 8.5 & 0.9 \\
\hline 20 & 12.8 & 8.5 & 6.4 & 5.1 & 4.3 & 0.4 \\
\hline
\end{tabular}


Description of MWD shape by asymmetry factor $\left(A_{s}\right)$, skewness $\left(\alpha_{3}\right)$ and kurtosis $\left(\alpha_{4}\right)$

The asymmetry factors $\left(A_{s}\right)$ of our MWDs were calculated using the ECOSEC Analysis program. $A_{\mathrm{s}}$ is defined as the ratio of the distance from the peak maximum to the right edge of the peak and the distance from the peak maximum to the left edge of the peak at $10 \%$ peak height. A graphical description is provided in figure S1.

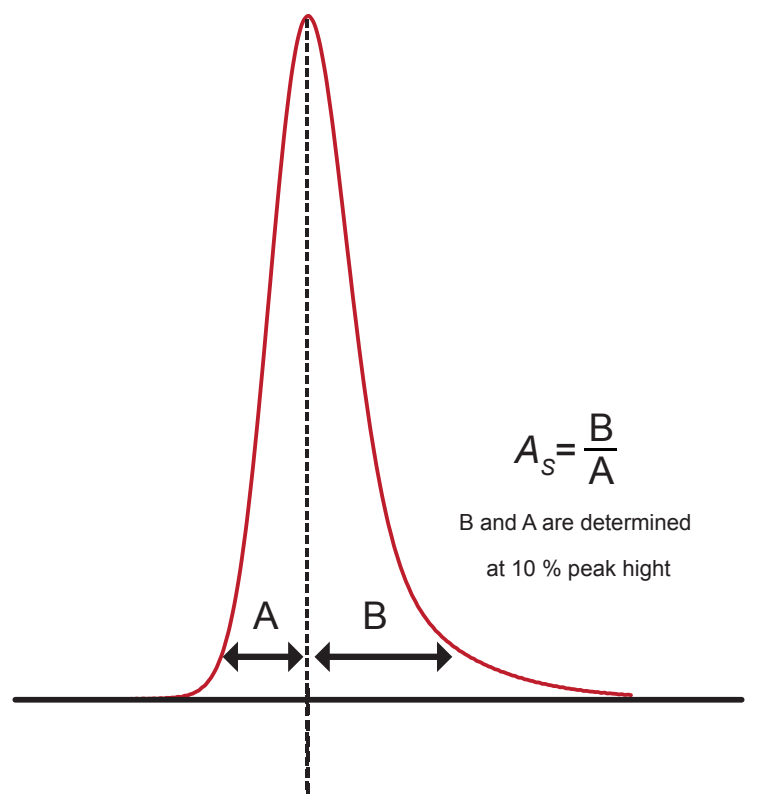

Figure S1. Graphical Illustration of the Calculation of Asymmetry Factor $A_{S}$

The third and fourth moments about the mean, skewness $\left(\alpha_{3}\right)$ and kurtosis $\left(\alpha_{4}\right)$, respectively, were calculated according to the method described by Rudin. ${ }^{1}$ The equations are shown below.

$$
\begin{gathered}
a_{3}=\frac{M_{z} M_{w} M_{n}-3 M_{n}^{2} M_{w}+2 M_{n}^{3}}{\left(M_{w} M_{n}-M_{n}^{2}\right)^{3 / 2}} \\
a_{4}=\frac{M_{z+1} M_{z} M_{w} M_{n}-4 M_{n}^{2} M_{z} M_{w}+6 M_{n}^{3} M_{w}-3 M_{n}^{4}}{\left(M_{w} M_{n}-M_{n}^{2}\right)^{2}}
\end{gathered}
$$


General Procedure for Poly(styrene-b-isoprene) (PS-b-PI) Polymerizations and Sample Preparation for Dynamic Mechanical Analysis

Polystyryllithium was prepared according to the general procedure. After approximately 5 hours, $1 \mathrm{~mL}(9.99 \mathrm{mmol})$ of isoprene was added to the stirring reaction mixture. The solution turned colorless. The reaction was allowed to stir for 6 hours to allow for full conversion according to ${ }^{1} \mathrm{H}$ NMR. See Table $\mathrm{S} 6$ for details on block polymer compositions.

Table S6: PS-b-PI samples for dynamic mechanical analysis; $f_{\mathrm{Pi}}$ indicates the weight fraction of polyisoprene

\begin{tabular}{|c|c|c|c|c|c|c|}
\hline entry & PS $M_{n}$ & PS $\oplus$ & PS $A_{s}$ & PSPI $M_{n}$ & PS-PI $\oplus$ & $f_{\mathrm{PI}}$ \\
\hline $1 a$ & 14.1 & 1.19 & 1.85 & 35.6 & 1.14 & 0.60 \\
\hline $1 b$ & 14.2 & 1.22 & 0.52 & 35.1 & 1.16 & 0.60 \\
\hline $2 a$ & 14.9 & 1.42 & 3.52 & 36.8 & 1.21 & 0.60 \\
\hline $2 b$ & 14.1 & 1.39 & 0.37 & 33.6 & 1.22 & 0.58 \\
\hline $3 a$ & 15.2 & 1.69 & 4.48 & 36.3 & 1.25 & 0.58 \\
\hline $3 b$ & 14.1 & 1.71 & 0.31 & 36.5 & 1.28 & 0.61 \\
\hline $4 a$ & 14.4 & 2.01 & 4.97 & 37.2 & 1.28 & 0.61 \\
\hline $4 b$ & 13.8 & 1.97 & 0.26 & 34.4 & 1.29 & 0.60 \\
\hline $5 a$ & 15.1 & 1.55 & 4.14 & 37.7 & 1.21 & 0.60 \\
\hline $5 b$ & 14.9 & 1.51 & 0.33 & 35.5 & 1.23 & 0.60 \\
\hline $6 a$ & 14.2 & 1.87 & 4.71 & 34.8 & 1.30 & 0.59 \\
\hline $6 b$ & 15.5 & 1.78 & 0.29 & 36.3 & 1.32 & 0.57 \\
\hline $7 a$ & 14.5 & 1.68 & 0.32 & 35.9 & 1.27 & 0.60 \\
\hline
\end{tabular}

\section{Preparation of Ps-b-PI samples for Dynamic Mechanical Analysis}

$0.2 \mathrm{wt} \%$ BHT as a stabilizer was added as a solution in DCM followed by concentrating on a rotary evaporator and vacuum overnight. After densifying at $120^{\circ} \mathrm{C}$ and $2000 \mathrm{lbs}$. for 5 min using a Carver Press, samples were compression molded into dog bones (16 $\mathrm{mm}, 2.5 \mathrm{~mm}, 0.6 \mathrm{~mm}$ ) at the same temperature and pressure for 5 minutes. 


\section{Measurement of Young's Moduli of PS-b-PI samples}

Stress/strain curves were obtained in tension with force control of $1.0 \mathrm{~N} / \mathrm{min}$ and Young's moduli were obtained by evaluating the slope of the stress/strain curve at low strain values.

Table S7. Complete polymer data from Figure 6.

\begin{tabular}{|c|c|c|c|c|c|c|}
\hline Entry & $\begin{array}{c}M_{\mathrm{n}}(\mathrm{S}) \\
(\mathrm{kg} / \mathrm{mol})\end{array}$ & $\oplus(\mathrm{S})$ & $A_{\mathrm{s}}$ & $\begin{array}{c}M_{\mathrm{n}}(\mathrm{SI}) \\
(\mathrm{kg} / \mathrm{mol})\end{array}$ & $\oplus(\mathrm{SI})$ & $\begin{array}{c}E \\
(\mathrm{MPa})\end{array}$ \\
\hline $1 \mathrm{a}$ & 14.1 & 1.19 & 1.85 & 35.6 & 1.14 & 460 \\
\hline $1 \mathrm{~b}$ & 14.2 & 1.22 & 0.52 & 35.1 & 1.16 & 560 \\
\hline $2 \mathrm{a}$ & 14.9 & 1.42 & 3.52 & 36.8 & 1.21 & 450 \\
\hline $2 \mathrm{~b}$ & 14.1 & 1.39 & 0.37 & 33.6 & 1.22 & 530 \\
\hline $3 \mathrm{a}$ & 15.2 & 1.69 & 4.48 & 36.3 & 1.25 & 220 \\
\hline $3 \mathrm{~b}$ & 14.1 & 1.71 & 0.31 & 36.5 & 1.28 & 440 \\
\hline $4 \mathrm{a}$ & 14.4 & 2.01 & 4.97 & 37.2 & 1.28 & 85 \\
\hline $4 \mathrm{~b}$ & 13.8 & 1.97 & 0.26 & 34.4 & 1.29 & 300 \\
\hline $5 \mathrm{a}$ & 15.1 & 1.55 & 4.14 & 37.7 & 1.21 & 310 \\
\hline $5 \mathrm{~b}$ & 14.9 & 1.51 & 0.33 & 35.5 & 1.23 & 450 \\
\hline $6 \mathrm{a}$ & 14.2 & 1.87 & 4.71 & 34.8 & 1.30 & 190 \\
\hline $6 \mathrm{~b}$ & 15.5 & 1.78 & 0.29 & 36.3 & 1.32 & 430 \\
\hline $7 \mathrm{a}$ & 14.5 & 1.68 & 0.32 & 35.9 & 1.27 & 420 \\
\hline
\end{tabular}



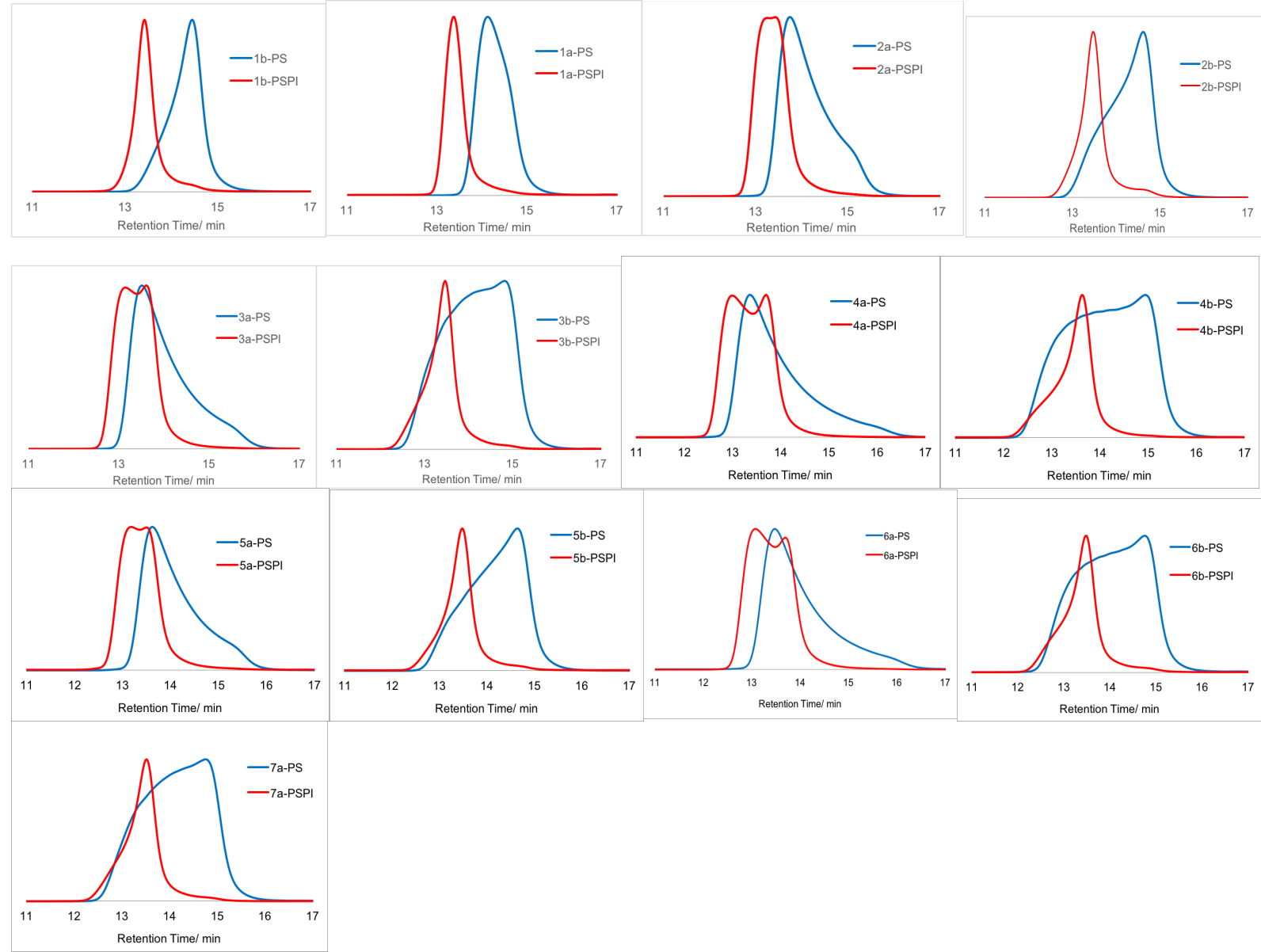

Figure S2. Size-exclusion chromatograms of polydisperse PS block (blue line) and the corresponding PS-b-PI block copolymers (red line).

Table S8. Comparison of light scattering and polystyrene standards for representative PS samples.

\begin{tabular}{|c|c|c|c|c|}
\hline & \multicolumn{2}{|c|}{ RI (PS standards) } & \multicolumn{2}{c|}{ LS } \\
\hline & $M_{\mathrm{n}}(\mathrm{kg} / \mathrm{mol})$ & $\oplus$ & $M_{\mathrm{n}}(\mathrm{kg} / \mathrm{mol})$ & $\oplus$ \\
\hline 40 min $\exp$ & 14.9 & 1.25 & 15.8 & 1.21 \\
\hline 60 min exp & 14.5 & 1.43 & 15.2 & 1.39 \\
\hline 40 min const & 15.8 & 1.41 & 16.4 & 1.39 \\
\hline
\end{tabular}




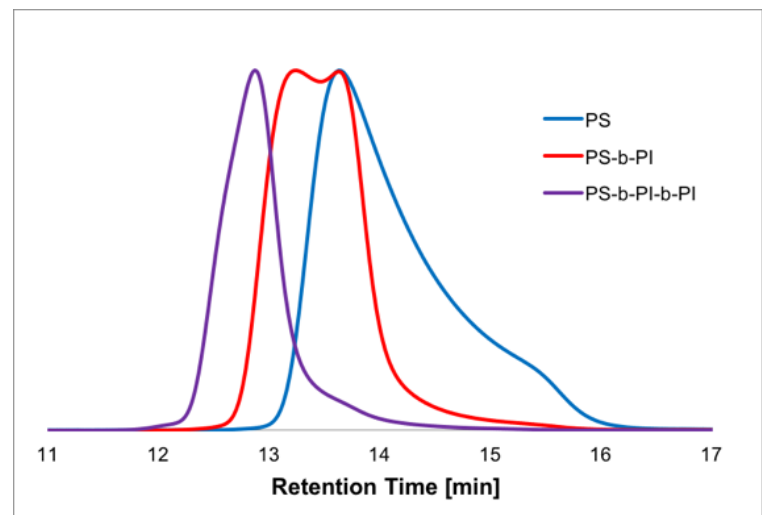

Figure S3. Chain-extension of a PS-b-PI compolymer with isoprene to give PS-bPI-b-PI copolymer. This experiment demonstrates that the bimodal distribution of these blocks is not due to chain termination.

\section{References:}

1. Rudin, A., J. Chem. Ed., 1969, 46, 595. 